EDITORIAL

\title{
Probióticos y aumento del peso corporal en humanos: conclusiones precipitadas y no basadas en la evidencia científica
}

\section{Do Probiotics Increase Body Weight in Humans? Hasty Conclusions and not Based on the Scientific Evidence}

\author{
Iva Marques-Lopes* \\ Facultad de Ciencias de la Salud y del Deporte (Huesca), Universidad de Zaragoza, Zaragoza, España
}

Recibido el 7 de septiembre de 2012; aceptado el 14 de septiembre de 2012

En mayo de 2012 apareció en los medios de comunicación masivos españoles una comunicación en la que se postulaba que la ingesta de probióticos podía suponer un riesgo para la salud de la población por agravar o fomentar uno de los problemas de salud pública más preocupantes en este momento: la obesidad ${ }^{1}$.

En septiembre de 2009, el investigador francés Didier Raoult realizó unos controvertidos comentarios en el editorial de la prestigiosa revista anglosaj ona Nature reviews: Microbiology, apoyando dichas afirmaciones en varios estudios realizados en humanos y en animales ${ }^{2}$. En diciembre del mismo año, tres investigadores (Dusko Ehrlich, Nathalie Delzenne y Gregor Reid), realizaron en la misma revista duras críticas a los comentarios realizados por Raoult ${ }^{3,4}$. Las principales líneas de crítica fueron ${ }^{3,4}$ : a) las hipótesis se han realizado sin una evaluación exhaustiva de la evidencia científica existente, con una interpretación exagerada de los datos de los estudios citados y omitiendo trabajos de la misma calidad científica con efectos neutros o contrarios; b) el uso de una lógica errónea en cuanto a las cepas de microorganismos implicados, y c) que el aumento de peso observado en los animales de granja tras la administración de probióticos respondía a un aumento de la masa magra debido a la mejora a la resistencia a las infecciones (y por lo tanto a menor cantidad de diarreas), y no al aumento de masa grasa debido al desequilibrio metabólico que causa la

\footnotetext{
*Autor para correspondencia.

Correo electrónico: imarques@unizar.es (I. Marques-Lopes).
}

obesidad. La controversia científica está, sin lugar a dudas, servida.

El 24 de mayo de 2012, en el número 2 de la revista Microbial Pathogenesis, el equipo de Didier Raoult publicó un metaanálisis ${ }^{5}$ que quizá fuera el detonante para volver al tema de debate. Sin embargo, en dicho metaanálisis existen varios errores, omisiones e irregularidades metodológicas que no pasan inadvertidas. Un ejemplo es la mezcla de estudios en humanos y animales en el análisis de los datos. Pese a que los propios autores incluyen este hecho en el apartado de limitaciones del estudio y que en el cuerpo del estudio se dice explícitamente que cuando se analizaron datos de estudios realizados solamente en humanos ningún resultado fue estadísticamente significativo, sus conclusiones finales no parecen verse modificadas en modo alguno. De nuevo, la controversia científica está servida.

Paradój icamente, el Comité Editorial de REVISTA ESPAÑOLA DE Nutrición Humana y Dietética recibió en diciembre de 2011 un artículo titulado "Efecto de los probióticos en el control de la obesidad en humanos: hipótesis no demostradas", que se incluye en el presente número. Los autores del artículo, después de una extensa revisión bibliográfica de estudios realizados en modelos celulares animales, pero también de estudios observacionales y ensayos clínicos en humanos, concluyen:

"Aunque se necesitan más estudios para evaluar los efectos de los probióticos en la incidencia de obesidad, si las relaciones establecidas entre la microbiota intestinal, el 
metabolismo y la inmunidad se demostraran mediante ensayos clínicos en humanos, el uso de estrategias dietéticas destinadas a modular la composición de la microbiota basadas en probióticos podría contribuir al control de los trastornos metabólicos de forma más eficaz y se abrirían nuevas puertas de estudio para obtener un mejor conocimiento de la biología humana y numerosas aplicaciones en la salud y la nutrición humana".

Es decir, que a la vez que aparecían alarmas sociales señalando a los probióticos como potenciales actores potenciadores de la obesidad, los científicos españoles señalaban, basándose en estudios científicos en humanos ${ }^{6-9}$, todo lo contrario: que el consumo de probióticos podría ser una nueva herramienta para modular la microbiota humana y ayudar así en la prevención de la obesidad. Los autores del artículo de revisión aciertan al incluir en el título la frase final: "hipótesis no demostradas".

Trabajar mediante la evidencia científica significa, en muchas ocasiones, tener que esperar a que haya más y mejores estudios para establecer una recomendación que sea altamente improbable que varíe con el tiempo ${ }^{10}$. La Práctica Dietética Basada en la Evidencia, definida en 2010 por la International Confederation of Dietetic Associations (ICDA) ${ }^{11}$, "[...] consiste en la búsqueda sistemática de evidencia científica y la evaluación de la validez, la aplicabilidad y la importancia de dicha evidencia para que, combinada después con la experiencia clínica del dietista-nutricionista, las opiniones y las circunstancias y valores específicos del cliente o la comunidad, sirva de guía en la toma de decisiones en el ámbito de la dietética".

Si bien parece existir una relación entre peso corporal y microbiota intestinal, según la evidencia científica y teniendo en cuenta los pocos estudios de intervención realizados en humanos, el carácter observacional de la mayoría de los estudios científicos disponibles y, por lo tanto, ante la imposibilidad de determinar si la alteración de la microbiota intestinal es causa o consecuencia de la modificación del peso corporal, no es de extrañar que dos grupos de in- vestigación distintos lleguen a hipótesis no demostradas contradictorias. Sin lugar a dudas, se requieren más y mejores ensayos clínicos controlados y aleatorizados en humanos para esclarecer las hipótesis generadas.

\section{Bibliografía}

1. Basulto J, Manera M, Baladia E. Postura del GREP-AEDN: la obesidad como enfermedad. Act Diet. 2008: 12:98-9.

2. Raoult D. Probiotics and obesity: a link? Nat Rev Microbiol. 2009 Sep; 7(9):616

3. Ehrlich SD. Probiotics - little evidence for a link to obesity. Nat Rev Microbiol. 2009; 7:901; author reply, 901.

4. Delzenne N, Reid G. No causal link between obesity and probiotics. Nat Rev Microbiol. 2009; 7:901; author reply, 901

5. Million M, Angelakis E, Paul M, Armougom F, Leibovici L, Raoult D. Comparative meta-analysis of the effect of Lactobacillus species on weight gain in humans and animals. Microb Pathog. 2012; 53:100-8

6. Santacruz A, Collado MC, Azcona C, et al. Weight loss influences gut microbial composition in 26 overweight adolescents. Obesity. 2009;23:1-10.

7. Santacruz A, Marcos A, Wärnberg J, Martí A, Martin-Matillas M, Campoy $C$, et al; EVASYON Study Group. Interplay between weight loss and gut microbiota composition in overweight adolescents. Obesity (Silver Spring). 2009;17:1906-1.

8. Turnbaugh $\mathrm{PJ}$, Hamady M, Yatsunenko T, Cantarel BL, Duncan $A$, Ley RE, et al. A core gut microbiome in obese and lean twins. Nature. 2009; 457:480-4.

9. Ilmonen J, Isolauri E, Poussa T, Laitinen K. Impact of dietary counselling and probiotic intervention on maternal anthropometric measurements during and after pregnancy: a randomized placebo-controlled trial. Clin Nutr. 2011;30:156-64.

10. Baladia E, Basulto J. Sistema de clasificación de los estudios en función de la evidencia científica. Dietética y Nutrición Aplicada Basadas en la Evidencia (DNABE): una herramienta para el dietista-nutricionista del futuro. Act Diet. 2008;12:11-9.

11. Evidence-based Practice Working Group of the International Confederation of Dietetic Associations (ICDA). Final Report of the International Confederation of Dietetic Associations (ICDA) Evidence-based Practice Working Group. November 25, 2010. 